\title{
PARTICIPANTS
}

TC Aitchison

Department of Statistics

University of Glasgow

University Gardens

Glasgow G12 8QW Scotland

Janet Ambers

British Museum Research Laboratory

Gt Russell Street

London WC1B 3DG UK

MGL Baillie

Palaeoecology Centre

Queen's University of Belfast

Belfast BT7 1NN No Ireland

Murdoch S Baxter

Scottish Universities Research

\& Reactor Centre

East Kilbride

Glasgow G75 OQU Scotland

Roelf Beukens

IsoTrace Laboratory

University of Toronto

Toronto ON M5S 1AZ Canada

Georges Bonani

Inst $\mathrm{f}$ Mittelenergie physik

ETH Honggerberg

CH 8093 Zürich Switzerland

Gilberto Calderoni

Dept Scienze della Terra

Universita di Roma

Piazzale Aldo Moro 5

00100 Rome Italy

Gordon Cook

Scottish Universities Research

\& Reactor Centre

East Kilbride

Glasgow G75 OQU Scotland
Jacques Evin

Radiocarbon Laboratory

Université Claude Bernard-Lyon I

43, Boulevard du 11 Novembre 1918

69622 Villeurbanne Lyon France

Adriano del Fabbro

Centro di Ricerca Applicata

Via pradamano 2/A

33100 Udine Italy

Marc Fournier

Laboratoire de Geochronologie

Centre de Bondy, ORSTOM

70-74 Route d'Aulnay

93140 Bondy France

José Garcia-Martinez

Radiocarbon Laboratory

Department de Quimica Analitica

Diagonal 647

University of Barcelona

Barcelona 28 Spain

Mebus Geyh

Niedersachsisches für Landesamt

und Bodenforschung

Postfach 510153

D-3000 Hannover-Bucholz W Germany

Roberto Gonfiantini

Isotope Hydrology Section

IAEA

PO Box 100

A-1400 Vienna Austria

Cecilio González-Gómez

Laboratorio de Datacion por Carbono 14

Facultad de Ciencias

Universidad de Granada

18071 Granada Spain

Steinar Gulliksen

Radiological Dating Laboratory

Norwegian Institute of Technology

N-7034 Trondheim NTH Norway 
DD Harkness

Scottish Universities Research

\& Reactor Centre

East Kilbride

Glasgow G12 OQU Scotland

Ede Hertelendi

Institute of Nuclear Research (ATOMKI)

Hungarian Academy of Sciences

Bem ter 18/C Pf51

Debrecen H4001 Hungary

Nada Horvatinčić

Rudjer Boskovic Institute

PO Box 1016

41000 Zagreb Yugoslavia

Högne Jungner

Radiocarbon Dating Laboratory

University of Helsinki

Snellmaninkatu 5

SF-00170 Helsinki Finland

Lauri Kaihola

Research Department

Wallac Oy

PO Box 10

SF-20101 Turku 10 Finland

Tuovi Kankainen

Geological Survey of Finland

SF-02150 Espoo Finland

Michael Kessler

Packard Instrument Co

One State St

Meridan, Connecticut 06450 USA

Renee Kra

Department of Geosciences

The University of Arizona

4717 E Ft Lowell Road

Tucson, Arizona 85712 USA

Jan Lanting

Biologish-Archaeologisch Inst

University of Groningen

Postraat 6

9712 ER Groningen The Netherlands
Austin Long

Department of Geosciences

The University of Arizona

Tucson, Arizona 85712 USA

Juan Mestres-Torres

Radiocarbon Laboratory

Department de Quimica Analitica

Diagonal 647

University of Barcelona

Barcelona 28 Spain

Brian F Miller

Scottish Universities Research

\& Reactor Centre

East Kilbride

Glasgow G75 OQU Scotland

Willem G Mook

Isotope Physics Lab

University of Groningen

Westersingel 34

9718 CM Groningen The Netherlands

John Noakes

CAIS

University of Georgia

120 Riverbend Road

Athens, Georgia 30605 USA

Ingrid Olsson

${ }^{14} \mathrm{C}$ Laboratory

Department of Physics

Uppsala University

Box 530

S-751 21 Uppsala Sweden

Barbara Ottaway

Department of Archaelogical Sciences

Bradford University

Bradford BD7 1DP UK

Mieczysław F Pazdur

Radiocarbon Laboratory

Institute of Physics

Silesian Technical University

Krzywoustego 2

PL-44-100 Gliwice Poland 
Gordon Pearson

Palaeoecology Centre

Queen's University of Belfast

Belfast BT7 1NN No Ireland

JR Pilcher

Palaeoecology Centre

Queen's University of Belfast

Belfast BT7 1NN No Ireland

\section{Henry Polach}

Radiocarbon Dating Research

Australian National University

GPO Box 4

Canberra ACT 2601 Australia

Mark Pollard

School of Chemistry and Applied

Chemistry

University of Wales

College of Cardiff

PO Box 912

Cardiff CF1 3TB Wales

Göran Possnert

Tandem Accelerator Laboratory

University of Uppsala

Box 533

S-75121 Uppsala Sweden

Jaan-Mati Punning

Institute of Ecology and Marine Research Academy of Sciences of the Estonian

Republic

1, Paldiski Road

200001 Tallinn Estonian Republic

Kasimierz Rozanski

Isotope Hydrology Section

IAEA

Wagramerstrasse 5

PO Box 100

A-1400 Vienna Austria
E Marian Scott

Department of Statistics

University of Glasgow

University Gardens

Glasgow G12 8QW, Scotland

Dr Göran Skog

Department of Quaternary Geology

Radiocarbon Laboratory

Tunavagen 29

S223 63 Lund, Sweden

Mrs F Smith

SERC SBAC

Polaris House

North Star Avenue

Swindon SN2 1ET, UK

Dr Donald Sutherland

2 London Street

Edinburgh EH3 6NA, Scotland

Dr Roy Switsur

Godwin Lab

Cambridge University

Free School Lane

Cambridge CB2 3RS, UK

Dr Mark van Strydonck

Koninklijk Inst voor Het

Kunstparti monium

Jupelpark 1

1040 Brussels, Belgium 